This document is published in:

Human Mutation, Vol. 32, no 11 (2011), pp. 1204-1212

DOI: http://dx.doi.org/10.1002/humu.21576

(C) 2011 Wiley Periodicals Inc. 


\title{
Kindler Syndrome: Extension of FERMT1 Mutational Spectrum and Natural History
}

\author{
Cristina Has, ${ }^{1}$ Daniele Castiglia, ${ }^{2}$ Marcela del Rio, ${ }^{3}$ Marta Garcia Diez, ${ }^{3}$ Eugenia Piccinni, ${ }^{2}$ Dimitra Kiritsi, ${ }^{1}$ Jürgen Kohlhase,${ }^{4}$ \\ Peter Itin, ${ }^{5}$ Ludovic Martin, ${ }^{6}$ Judith Fischer, ${ }^{7}$ Giovanna Zambruno, ${ }^{2}$ and Leena Bruckner-Tuderman ${ }^{1,8 *}$ \\ ${ }^{1}$ Department of Dermatology, University Medical Center Freiburg, Freiburg, Germany; ${ }^{2}$ Laboratory of Molecular and Cell Biology, Istituto \\ Dermopatico dell'Immacolata-IRCCS, Rome, Italy; ${ }^{3}$ CIEMAT-CIBER on Rare Diseases, Regenerative Medicine Unit-U714, Madrid, Spain; ${ }^{4}$ Center \\ for Human Genetics Freiburg, Freiburg, Germany, ${ }^{5}$ Department of Dermatology, University Hospital Basel, Basel, Switzerland; ${ }^{6}$ Department of \\ Dermatology, Angers University Hospital, Angers, France, ${ }^{7}$ Institute for Human Genetics, University Medical Center Freiburg, Freiburg, Germany; \\ ${ }^{8}$ Freiburg Institute for Advanced Studies, University of Freiburg, Freiburg, Germany
}

\begin{abstract}
Mutations in the FERMT1 gene (also known as KIND1), encoding the focal adhesion protein kindlin1 , underlie the Kindler syndrome (KS), an autosomal recessive skin disorder with an intriguing progressive phenotype comprising skin blistering, photosensitivity, progressive poikiloderma with extensive skin atrophy, and propensity to skin cancer. Herein we review the clinical and genetic data of 62 patients, and delineate the natural history of the disorder, for example, age at onset of symptoms, or risk of malignancy. Although most mutations are predicted to lead to premature termination of translation, and to loss of kindlin-1 function, significant clinical variability is observed among patients. There is an association of FERMT1 missense and in-frame deletion mutations with milder disease phenotypes, and later onset of complications. Nevertheless, the clinical variability is not fully explained by genotype-phenotype correlations. Environmental factors and yet unidentified modifiers may play a role. Better understanding of the molecular pathogenesis of KS should enable the development of prevention strategies for disease complications.
\end{abstract}

KEY WORDS: FERMT1; epidermolysis bullosa; kindlin; poikiloderma; blister; fermitin

\section{Background}

Kindler syndrome (KS; MIM\# 173650) is caused by mutations in FERMT1 (also known as KIND1; MIM\# 607900), the gene encoding kindlin-1 [Fine et al., 2008; Jobard et al., 2003]. KS is a

\footnotetext{
*Correspondence to: Leena Bruckner-Tuderman, Department of Dermatology, University Medical Center Freiburg, Hauptstr. 7, 79104 Freiburg, Germany. E-mail: brucknertuderman@uniklinik-freiburg.de

Contract grant sponsors: International Kindler Syndrome; The German Federal Ministry for Education and Research; The Excellence Initiative of the German federal and stage government and Freiburg Institute for Advanced Studies, School of Life Sciences (to L.B.T); The Italian Ministry of Heath.
}

rare autosomal recessive skin disorder with an intriguing progressive phenotype comprising skin blistering, photosensitivity, progressive poikiloderma, and extensive skin atrophy [Lai-Cheong and McGrath, 2010]. Additional clinical features include gingival erosions, ocular, esophageal, gastrointestinal and urogenital involvement, and an increased risk of mucocutaneous malignancy [Lai-Cheong and McGrath, 2010]. To date, about $150 \mathrm{KS}$ patients with FERMT1 mutations have been described in the literature. All 46 mutations reported so far are predicted to lead to premature termination of translation and to loss of the kindlin-1 protein or of its function [Lai-Cheong and McGrath, 2010; Techanukul et al., 2011]. The clinical and genetic aspects of the disease have recently been reviewed [Lai-Cheong and McGrath, 2010; Lai-Cheong et al., 2009], but precise data on genotype-phenotype correlations and the natural history of the disorder, for example, age at onset of symptoms, or risk of malignancy, still remain elusive.

The FERMT1 gene product, kindlin-1, is a member of the new protein family of kindlins-1, -2 , and -3. It is an epithelial-specific protein expressed in the skin, periodontal tissues, and colon [Herz et al., 2006; Kern et al., 2007; Petricca et al., 2009; Ussar et al., 2008]. In the skin, kindlin-1 is localized in basal epidermal keratinocytes and exists in two forms, nonphosphorylated and phosphorylated [Herz et al., 2006]. The four point one ezrin radixin and moesin (FERM) and pleckstrin homology (PH) domains are involved in molecular interactions, providing kindlin-1 the role of a focal adhesion adaptor protein linking filamentous actin in the cell cortex, to membrane proteins [Goult et al., 2009; Larjava et al., 2008]. In KS skin, disorganized keratinocytes lose their proper architecture, polarization, and the boundary to the dermis and exhibit minimal proliferation. In vitro, loss of kindlin-1 function is associated with abnormal cell shape and, functionally, cell adhesion, spreading, proliferation, and directed motility are perturbed [Herz et al., 2006; Petricca et al., 2009; Ussar et al., 2008]. These deficits stem from impaired functions of focal adhesions (FA), which assure the anchorage of the cytoskeleton to integrin-associated signaling platforms. Kindlin-1 is a member of the intracellular B1 integrin-associated signalling complex and, thus, of FA, but the function of kindlins as activators of $\beta$ integrins together with talin is still controversial [Harburger and Calderwood, 2009; Moser et al., 2009]. Whereas FA and $\beta 1$ integrin functions have been extensively studied in cultured cells, their significance and functionality in vivo in the skin and mucosa remain unclear. KS is the first FA skin disorder known so far and, therefore, represents a genetic model to understand the role of FA protein complexes in tissues in vivo. 

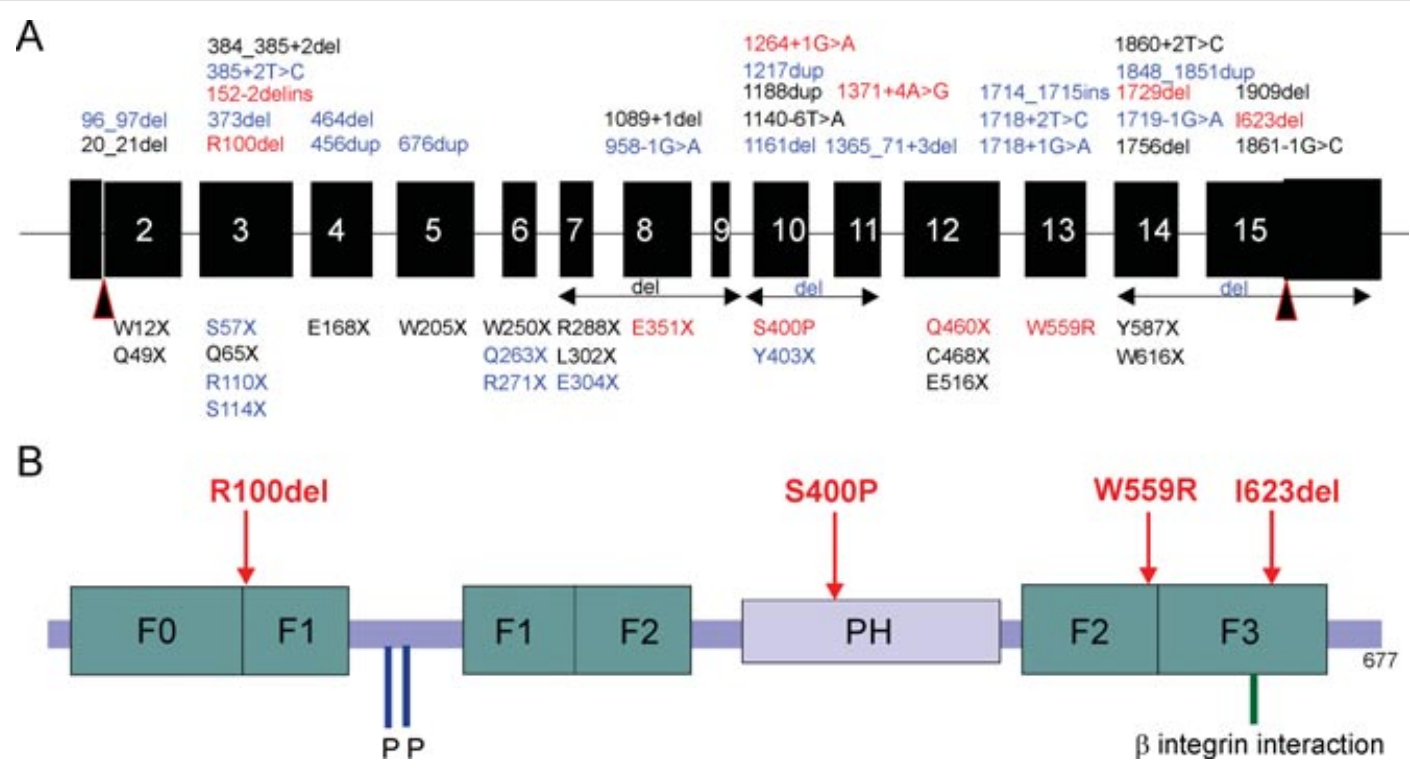

Figure 1. FERMT1 gene, kindlin-1 protein, and the spectrum of mutations. A: Schematic representation of the FERMT1 gene comprising 15 exons (black boxes). The mutations described in this study -in red, novel mutations and in blue, known mutations-and in the literature (in black) are depicted. B: The kindlin-1 protein with four point one ezrin radixin and moesin (F) and pleckstrin homology domains, phosphorylated serine residues and $\beta$ integrin binding region is shown. Note the location of the novel missense mutations and in-frame deletions reported in this study.

Here we review the FERMT1 mutational database and extend it with 10 novel mutations, including the first FERMT1 missense and in-frame deletion mutations associated with KS. Moreover, we evaluate the progressive phenotype and complications of KS in a cohort comprising 62 patients with defined FERMT1 mutations. To the best of our knowledge, this is the largest cohort systematically analyzd so far.

\section{Variants in the FERMT1 Gene}

All FERMT1 sequence changes published thus far, together with novel variants characterized in our laboratories, are represented in Figure 1A and in the Supp. Table S1. The genotypes and phenotypes of 62 patients included in the study are listed in Table 1.

The mutational analysis of $\mathrm{KS}$ in the present cohort was performed as described [Has et al., 2006]. Briefly, genomic DNA extracted from peripheral lymphocytes was used for PCR amplification of the entire coding region and exon-intron boundaries of the FERMT1 gene [Has et al., 2006]. The PCR products were processed for automated nucleotide sequencing in an ABI 3130XL genetic analyzer (ABI, Darmstadt, Germany). DNA sequences were compared to the reference sequences from the NCBI Entrez Nucleotide database (NM_017671.4; AL118505.17). Nucleotide numbering reflects cDNA numbering with +1 corresponding to the A of the ATG translation initiation codon in the reference sequence (www.hgvs.org/mutnomen). The initiation codon is codon 1 . The sequence variants were confirmed by resequencing of PCR products obtained from a second amplification reaction. For the novel allelic variants, at least 100 (for missense mutations 200) control chromosomes were screened by direct sequencing. Prediction of splice sites was performed with NetGene2 (http://www.cbs.dtu.dk/services/NetGene2/), whereas for prediction of the consequences of amino acid substitutions and single amino acid deletions, PolyPhen (http://genetics.bwh.harvard .edu/pph/) and Mutation Taster (http://neurocore.charite.de/
MutationTaster/index.html) were employed. The effects of the c. $1371+4 \mathrm{~A}>\mathrm{G}$ splicing mutation were also tested by reverse transcriptase (RT)-PCR analysis of the total RNA extracted from cultured keratinocytes of patient 4 , using primers and PCR conditions reported previously [Has et al., 2006].

This mutational survey disclosed 34 distinct FERMT1 sequence variants: 10 deletions, five duplications or insertions, one insertion or deletion, seven splice-site mutations, nine nonsense mutations, and two missense mutations (Fig. 1A; Table 1). Ten previously unknown mutations were found, including two nonsense mutations, two splice-site mutations, one deletion or insertion, three small deletions, and two missense mutations.

The novel mutations p.Glu351X and p.Gln460X increase the percentage of nonsense mutations in the FERMT1 mutational database to $37.5 \%$ (21 of 56). The c.1729del (p.Ser577AlafsX14) mutation results in a premature stop codon that deletes most of the FERM 3 domain of kindlin-1 or, more likely, triggers mRNA degradation via nonsense-mediated decay mechanisms. The splice-site mutation $c .1264+1 \mathrm{G}>\mathrm{A}$ changes the obligate $\mathrm{G}$ nucleotide of the splice donor site of exon 10 , whereas c. $1371+4 A>G$ is predicted to abolish the splice donor site of exon 11 (NetGene2). Consequently, exon 11 could be skipped, or an upstream cryptic splice site located in exon 11 (TGgtatga) could be activated leading to skipping of $32 \mathrm{nu}-$ cleotides; RT-PCR analysis and sequencing of the resulting cDNA product confirmed the latter prediction (not shown), which results in a frame shift and premature termination of translation. The deletion insertion c.152-2delAGinsCT mutates the invariant AG dinucleotide of the splice acceptor site of exon 3 and, therefore, interferes with the splicing process.

Here we defined the first FERMT1 in-frame deletions: p.Arg100del and p.Ile623del. Both were excluded in normal control chromosomes and were predicted to be disease relevant (Mutation Taster). Arginine 100, located within the FERM 1 subdomain (Fig. 1B), is conserved in mammalians, chicken, and Xenopus laevis. Although the precise consequence of this mutation remains to be established, the $\mathrm{N}$-terminal region of kindlins is believed to be 


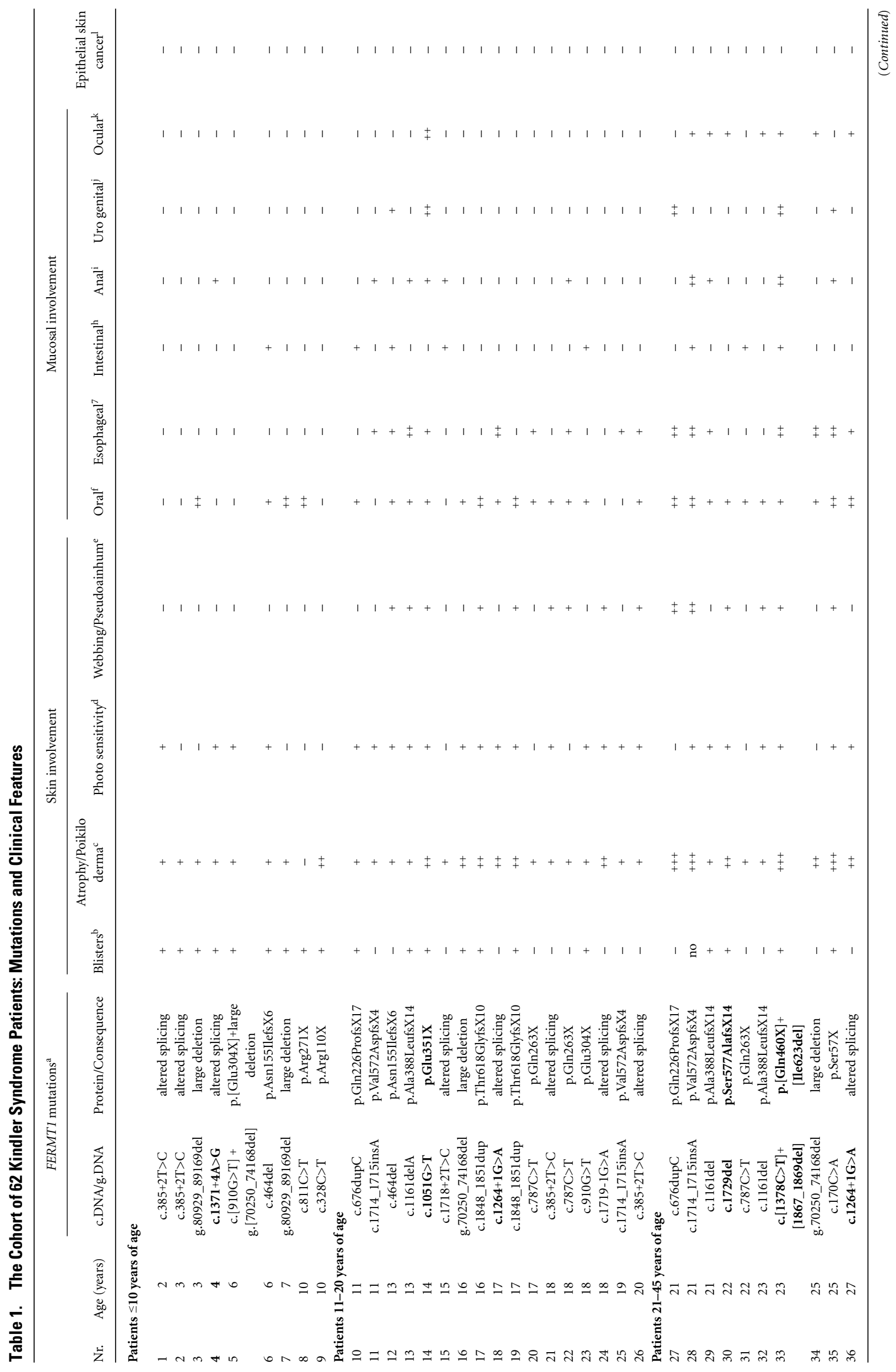




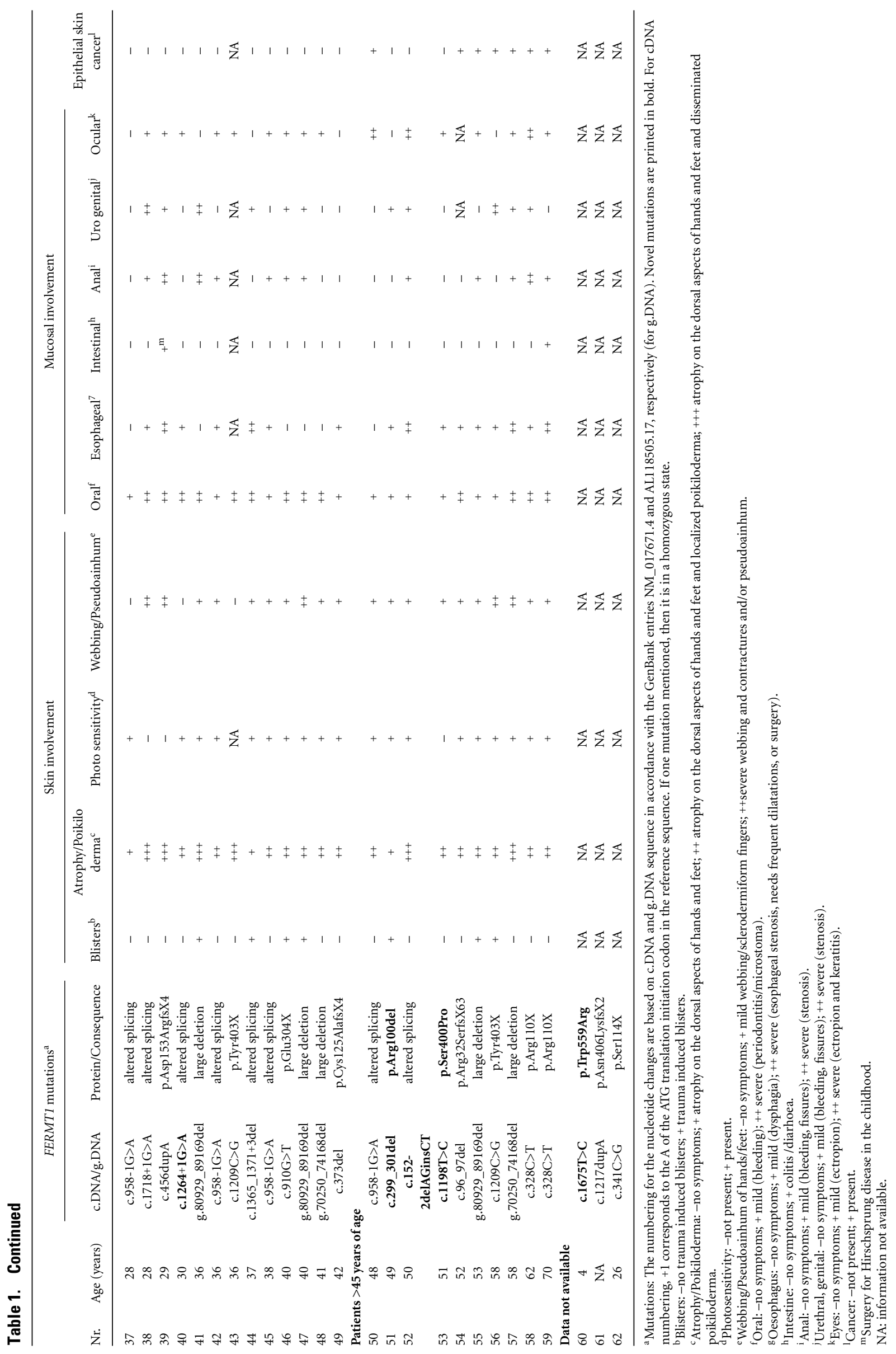


important for interaction with binding partners, such as integrins, migfilin, and integrin linked kinase [Goult et al., 2009; Larjava et al., 2008]. The in-frame deletion of isoleucine 623 affects the FERM 3 subdomain close to the binding site for $\beta 1$ integrin [Harburger et al., 2009] (Fig. 1B).

Importantly, we also report the first amino acid substitutions associated with a KS phenotype: p.Ser400Pro and p.Trp559Arg. Both mutations were excluded in 200 control chromosomes and were predicted to be disease causing by both Polyphen and Mutation Taster. However, the molecular consequences of these amino acid substitutions remain to be established. Serine 400 is conserved in mammalians, chicken, X. laevis, and in human kindlin-2, and it is located in the $\mathrm{PH}$ domain, which is considered to be important for protein-protein interactions (Fig. 1B). Tryptophan 559 within the FERM 2 domain is widely conserved among different species (Fig. 1B). The mutation replaces tryptophan, a nonpolar neutral amino acid, with arginine, which is polar and positively charged. This substitution is likely to perturb protein folding of the FERM domain and thus affect its function as the binding ligand for actin cytoskeleton.

\section{Genotype-Phenotype Correlation and Natural History of KS}

Sixty-two patients, 32 females and 30 males, originating from different parts of the world were included in this survey. Ten patients were $\leq 10$ years of age, 17 between 11 and 20 years of age, 24 between 21 and 45 years of age, and 10 were $>45$ years of age at the time of recording the clinical data. In three cases, no detailed information was available. The diagnosis was suggested by clinical features, history, and morphological analyses of the skin, and confirmed by disclosure of FERMT1 mutations. To assess the severity and the course of the disorder, the following clinical parameters were recorded: skin blistering, photosensitivity, skin atrophy, and poikiloderma, webbing of fingers and toes, pseudoainhum, extent of mucosal involvement, and development of epithelial skin cancers (Table 1).

\section{Genotype-Phenotype Correlation}

Thus far, two aspects have impeded assessment of genotypephenotype correlations in KS. First, because all previously reported mutations were predicted to be null mutations leading to loss of kindlin-1 and its functions, phenotypic variations could not be explained by the nature or the position of the mutation. The present study describing the first FERMT1 missense mutations and in-frame deletions enables the first true genotype-phenotype correlations in KS. Patient 51, homozygous for p.Arg100del, and patient 53, homozygous for p.Ser400Pro, are mildly affected and have not developed epithelial skin cancer, in spite of both being in their fifties. This suggests that mutations compatible with expression of mutated kindlin-1 protein will translate into mild phenotypes, whereas null mutations cause severe disease.

Second, the KS phenotype is progressive, evolving with age. Consequently, the severity of the symptoms in patients of different ages cannot be compared. Although all individuals with KS caused by null mutations exhibit skin blistering, poikiloderma, and photosensitivity, the variability of clinical presentation is evident, suggesting a role for environmental and/or epigenetic factors. We have recently shown that the cumulative effect of cell stress-inducing events may play a decisive role in the outcome of the disease [He et al., 2011; Heinemann et al., 2011]. Kindlin-1 deficient keratinocytes respond to cell stress by upregulating the expression of cytokines, for example, IL-20, IL-24, and TGF- $\beta 2$. An inflammatory response and activation of fibroblasts and their differentiation to myofibroblasts in the dermis are initiated. These data suggest that repeated cycles of epidermal cell stress, cytokine secretion, dermal inflammation, and profibrotic processes underlie mucocutaneous fibrosis in KS [Heinemann et al., 2011]. Skin and mucous membranes, which form the barrier to the environment, are permanently exposed to mechanical factors, UV irradiation, and pathogens. Increased environmental noxae may explain the extent of symptoms in severely affected KS patients described before [Has, et al. 2008a; Kern et al., 2007; Mansur et al., 2007].

\section{Natural History of KS}

Systematic analysis of the clinical features in 59 patients revealed the chronology of the onset of symptoms and complications in KS and allowed delineation of the natural history of the disorder in this cohort (Fig. 2; Table 1). Skin blistering was present in all (100\%) KS patients younger than 10 years (Fig. 3A), but progressively decreased with age. Skin fragility was considered relatively mild in the adult KS patients, since some of them reported not to get blisters even after using adhesive tapes. Photosensitivity was assessed subjectively by patients or parents. Seventy-six percent (45 of 59) reported mild photosensitivity, but 24\% (14 of 59) denied having problems with sunlight. Skin atrophy of the dorsal aspects of the hands was the first symptom indicative of KS in children, and was recognized as early as at the age of 2 years (Fig. 3B and C). Poikiloderma was first noted around the age of 10 years, and it progressed with age (Fig. 3D and E). Sclerosing features of the hands and feet, such as webbing, or pseudoainhum occurred only after the age of 10 years, but were present already in 53\% (9 of 17) of patients in the age group of 11-20 years. However, the severity seemed not to be exclusively agedependent. Cumulative mechanical stress may play an important role. For example, patient 27 who was treated during her childhood with tight bandages for her acral blisters had severe webbing and sclerotic fingers. In the present cohort, epithelial skin cancer was not diagnosed before the age of 45 years, but affected $70 \%$ ( 7 of 10) of the patients older than 45 years. All patients developed squamous cell carcinomas or precursor lesions, whereas basal cell carcinomas were occasionally diagnosed.

Mucosal involvement is very common in KS. The oral mucosa was most frequently affected, as observed in $85 \%$ (49 of 58) of the patients. Symptoms related to esophageal affection were not noted before the age of 10 years. Thereafter, they developed progressively evolving from dysphagia to strictures requiring repeated dilatations, affecting $53 \%, 67 \%$, and $89 \%$, of the patients in the age categories of $<20,21-45$, and $>45$ years, respectively (Fig. 2 ). Anal, urogenital, and ocular mucosa involvement occurred occasionally in adult patients (Fig. 2). Intestinal involvement, that is, colitis induced by epitheliolysis [Kern et al., 2007], was diagnosed in only 15\% (9 of 58) cases. This is in contrast to the kindlin-1 knockout mouse, which exhibited lethal intestinal involvement and a very mild skin phenotype [Ussar et al., 2008].

\section{Clinical Relevance}

Knowledge of the natural history enables better and early recognition of KS, and design of a prevention plan through adaptation of the life style (e.g. elimination of UV exposure, skin care). In KS, the phenotype evolves with improvement of skin blistering with advancing age, similar to epidermolysis bullosa simplex 


\section{Skin involvement}
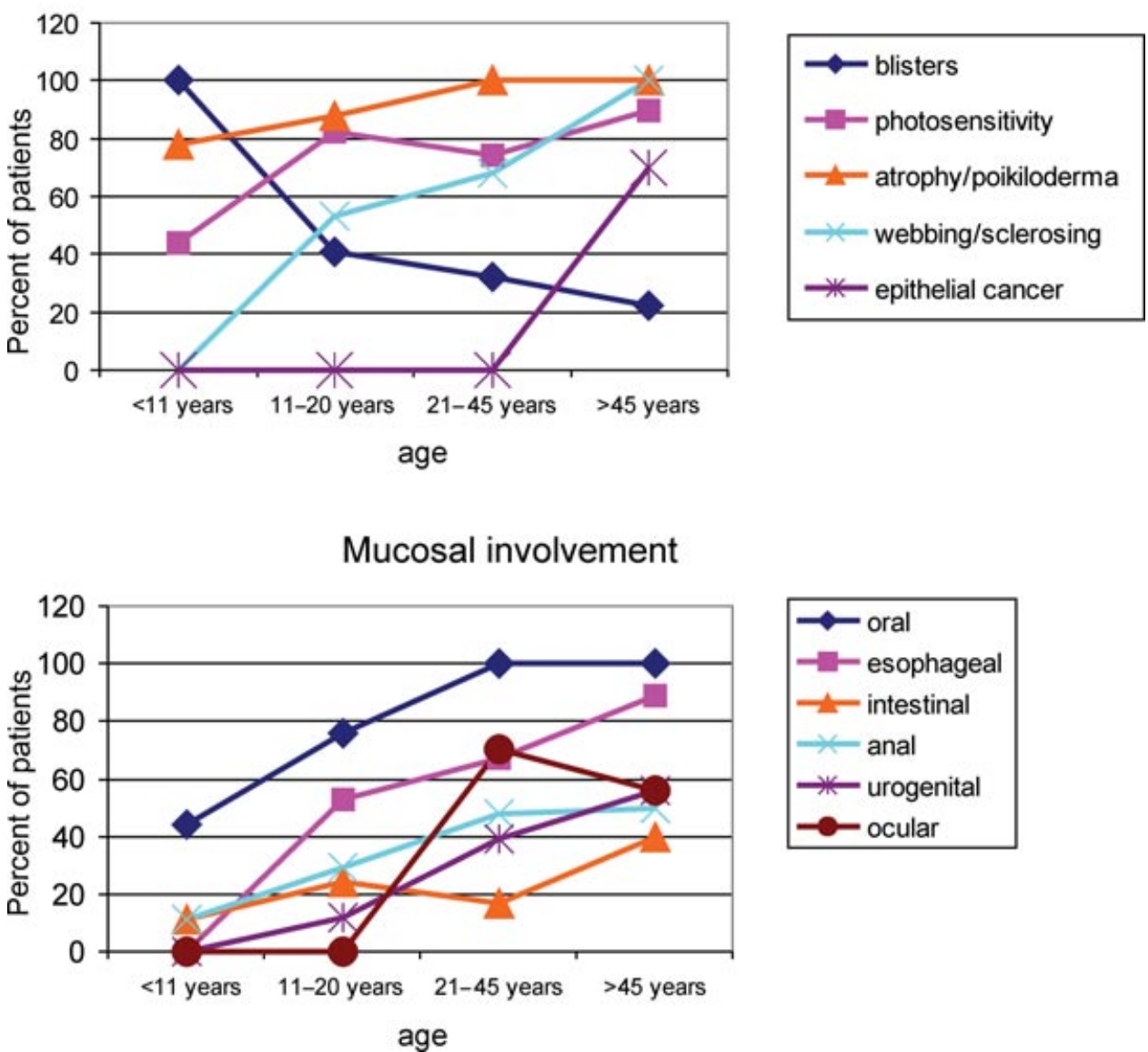

age

Figure 2. Natural history of Kindler syndrome (KS) in the present cohort. The upper graph shows the age-dependent course of skin involvement comprising skin blistering, photosensitivity, atrophy/poikiloderma, webbing/sclerotic changes of hands and feet, and epithelial skin cancer. The lower graph depicts the age-dependent course of the mucosal involvement. [Color figure can be viewed in the online issue, which is available at wiley.com/humanmutation.]

(Sprecher, 2010). There is no molecular explanation for this phenomenon, but several hypotheses can be discussed. First, FA are not major adhesive structures at the dermal-epidermal junction. Therefore, compensatory mechanisms involving other supramolecular adhesive structures, for example, hemidesmosomes could explain the amelioration of skin fragility. Second, with advancing age patients learn to adapt their lifestyle (e.g. to avoid exposure to mechanical stress and sun, constantly apply intensive skin care), and thus influence epigenetic effects. Third, based on personal observations, somatic genetic events may modify the course of the disease. Revertant mosaicism refers to the occurrence of revertant or second site mutations, which attenuate or abolish the effect of the disease-causing mutations, thus leading to improvement of the skin function.

Importantly, involvement of esophageal and genitourinary mucosa is common, and clearly increases with age. The same applies to the risk of nonmelanoma skin cancers. Almost all patients included in this study developed premalignant and malignant skin lesions after the age of 45 years, whereas in the general population, the incidence rates for squamous cell carcinoma are 4.8/100,000 in men and $0.6 / 100,000$ in women, in the same age group [Stang et al., 2003]. Early diagnosis and treatment of such conditions are essential for prevention of severe progression [Arita et al., 2007; Emanuel et al., 2006].
Noteworthy, a broad spectrum of differential diagnoses had been considered in our patients, including dystrophic epidermolysis bullosa, epidermolysis bullosa simplex, xeroderma pigmentosum, and systemic scleroderma. Conversely, patients with particular forms of junctional epidermolysis bullosa, and EBS with mottled pigmentation were suspected to suffer from KS, because of substantial overlap in clinical and morphologic findings.

Importantly, the majority of KS patients reported thus far are homozygous for their mutations. They are predominantly offspring of consanguineous marriages, or originate from isolated populations (e.g. Native Americans from Panama [Penagos et al., 2004; Siegel et al., 2003], immigrants in Brazil [Martignago et al., 2007]). Some mutations seem to affect hot spots or to have occurred long time ago, being disseminated in populations (e.g. g.70250_74168del in southern Italy [Has et al., 2006], g.80929_89169del in the Balkans, c.676dup in the former Yugoslavia reported here, Table 1). It is difficult to assess the global incidence of KS. Thus far, about 150 patients have been reported in the literature, but the number of affected persons is certainly higher, because KS is often misdiagnosed and underestimated.

Mutation analysis remains the gold standard of KS diagnosis. However, since skin blistering is a dominant symptom in childhood-for this reason KS is considered a subtype of epidermolysis bullosa-morphological analysis of a skin biopsy is 

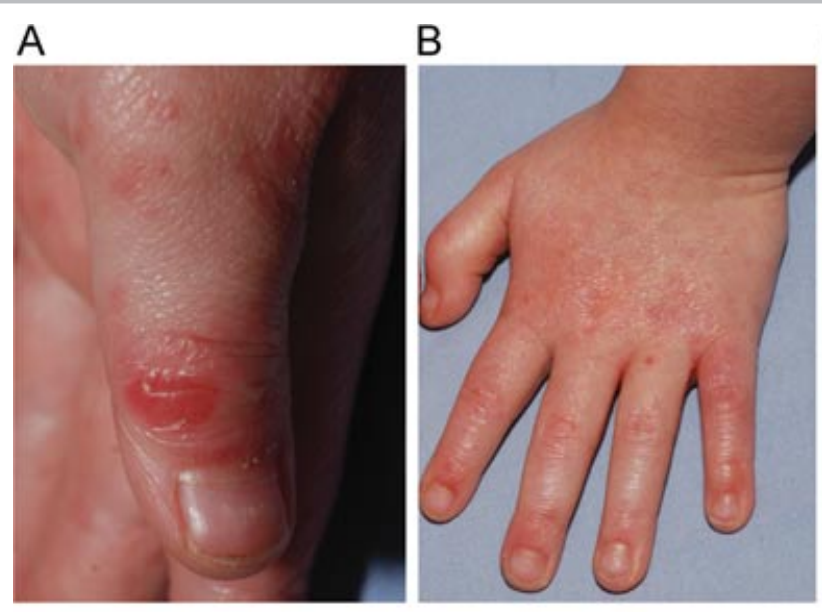

C
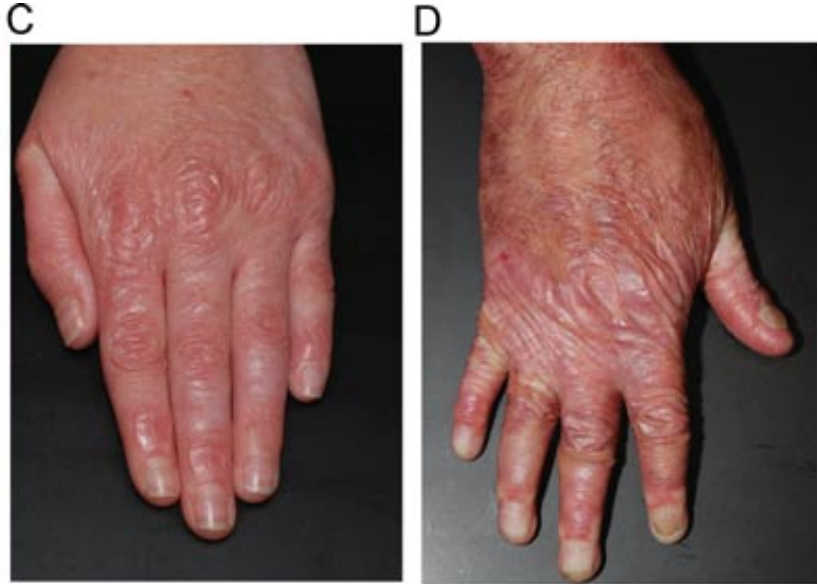

$\mathrm{E}$

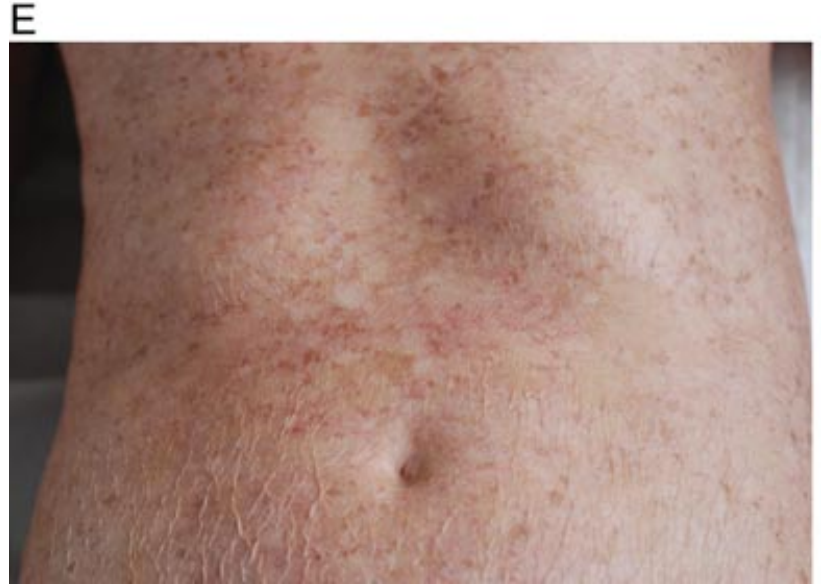

$\mathrm{F}$

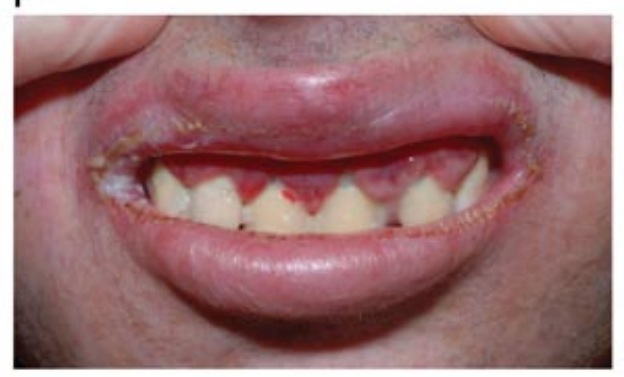

Figure 3. Clinical features of KS. A: Acral blisters in a 2-year-old patient. B: Incipient skin atrophy in the same patient. C: Pronounced atrophy on the dorsal aspects of the hands in an 8-year-old patient. D: Poikiloderma, cigarette-paper-like atrophy, and webbing of the fingers at the age of 28 years. E: Generalized poikiloderma in a 53-year-old patient. F: Severe involvement of the oral mucosa in a 28 -year-old KS patient.

often the first diagnostic procedure performed. The indirect immunofluorescence (IIF) and transmission electron microscopy (TEM) findings of irregular, branched, and interrupted basement membrane are characteristic and indicative of KS and justify FERMT1 mutation analysis for confirmation of the suspected diagnosis.

Here, in the majority of cases, IIF staining was performed to support the genetic diagnosis and explore the consequences of kindlin-1 mutations on the morphology of the skin, in particular of the dermal-epidermal basement membrane. IIF staining was performed on 5- $\mu \mathrm{m}$ cryosections of KS and normal skin. Skin sections were blocked with $0.1 \% \mathrm{BSA} / \mathrm{TBS}$ for $30 \mathrm{~min}$ and treated with primary antibodies to collagen IV (Col IV-22, Abcam), collagen XVII [Hofmann et al., 2009], pankeratin (Dako, Hamburg, Germany), BM165 to human laminin $\alpha 3$ chain (a gift from Dr. P. Rousselle, Lyon, France), $6 \mathrm{~F} 12$ to human laminin $\beta 3$ chain (a gift from Dr. RE Burgeson, Cutaneous Biology Research Center, Harvard Medical School, Cambridge, MA), GB3 to laminin $\gamma 2$ chain (a gift of Dr. G. Meneguzzi, Nice, France), and LH 7.2 to the NC1-domain of collagen VII (Sigma, Taufkirchen, Germany), and kindlin-1 [Herz, et al., 2006]. The secondary antibodies were fluorescein isothiocyanate-labelled anti-mouse or anti-rabbit IgG. The signals were visualized with epifluorescence microscopy (Zeiss Axio Imager, Zeiss). Typically, thickened and interrupted basement mem- brane labelling for laminin-332, collagen IV and collagen VII was observed (Fig. 4A). In some cases, kindlin-1 staining was performed and revealed absence of a signal at the dermal-epidermal junction, although the polyclonal sera give a background staining in the entire epidermis (Fig. 4B).

As morphologic features at light microscopic level, epidermal atrophy and microblisters at the dermal-epidermal junction were noted. In five cases, TEM demonstrated focal widening of the lamina lucida (microdetachments), reduplications of the lamina densa and, occasionally, moderately hypoplastic hemidesmosomes.

FERMT1 mutations are found in patients with typical clinical findings of KS and/or typical morphological aspect in IIF or TEM. In our experience, in cases without FERMT1 mutations, clinical and skin morphological data are usually lacking, making the diagnosis rather difficult. Moreover, most antibodies to kindlin-1 give high levels of background, and therefore, loss of the expression of the protein cannot be reliably established by IIF of skin samples. In cases with strong suspicion of $\mathrm{KS}$, in whom no FERMT1 mutations are disclosed by direct sequencing, and large deletions are excluded, extensive analyses of RNA and proteins extracted from cultivated keratinocytes should shed light onto the molecular disease mechanisms. However, the evaluation of the pathogenic role of missense mutations requires further investigations. 

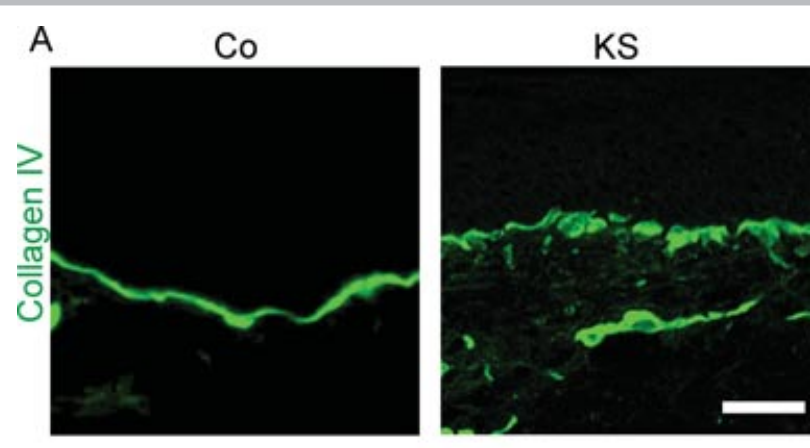

B
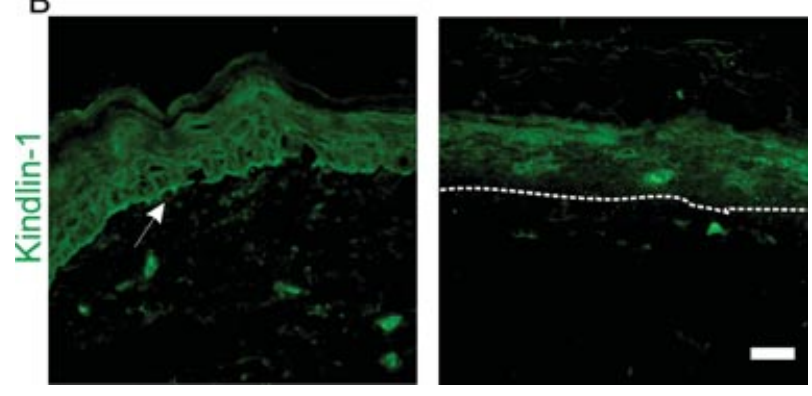

Figure 4. Morphological abnormalities of KS skin. Immunofluorescence staining of control and KS skin with antibodies to collagen IV and kindlin-1. A: In contrast to the linear deposition of collagen IV at the epidermal basement membrane in control skin, an irregular and interrupted staining pattern is seen in KS skin. B: Note the linear localization of kindlin-1 at the dermal-epidermal junction in control skin (arrow), and its absence in KS skin (the interrupted line depicts the dermalepidermal junction). The antibody gives a background staining in the entire epidermis.

\section{Future Prospects}

Future research will be directed toward better understanding of molecular disease mechanisms, including genotype-phenotype correlations and modifiers of the phenotype, making use of mouse models, recombinant systems, or proteomics approaches. The consequences of missense mutations and in-frame deletions, which seem to account for milder phenotypes, remain to be elucidated. Prevention of complications and treatment prospects might arise from identification of modifying factors. A further goal is the clinical and genetic delineation of KS-like phenotypes, which are not caused by FERMT1 mutations. In such cases, identification of new disease-causing genes will be possible by whole-exome sequencing in nuclear families [Glazov et al., 2011].

A locus-specific mutation database is available at: http:// www.LOVD.nl/FERMT1.

\section{Acknowledgments}

We thank all patients who participated in this study and the physicians who sent us samples. The excellent technical assistance by Vera Morand, Margit Schubert, and Kaethe Thoma is gratefully acknowledged.

\section{References}

Arita K, Wessagowit V, Inamadar AC, Palit A, Fassihi H, Lai-Cheong JE, Pourreyron C, South AP, McGrath JA. 2007. Unusual molecular findings in Kindler syndrome. Br J Dermatol 157:1252-1256.
Ashton GH, McLean WH, South AP, Oyama N, Smith FJ, Al-Suwaid R, Al-Ismaily A, Atherton DJ, Harwood CA, Leigh IM, Moss C, Didona B, Zambruno G, Patrizi A, Eady RA, McGrath JA. 2004. Recurrent mutations in kindlin-1, a novel keratinocyte focal contact protein, in the autosomal recessive skin fragility and photosensitivity disorder, Kindler syndrome. J Invest Dermatol 122:78-83.

Burch JM, Fassihi H, Jones CA, Mengshol SC, Fitzpatrick JE, McGrath JA. 2006. Kindler syndrome: a new mutation and new diagnostic possibilities. Arch Dermatol 142:620-624.

Emanuel PO, Rudikoff D, Phelps RG. 2006. Aggressive squamous cell carcinoma in Kindler syndrome. SKINmed 5:305-307.

Fassihi H, Wessagowit V, Jones C, Dopping-Hepenstal P, Denyer J, Mellerio JE, Clark S, McGrath JA. 2005. Neonatal diagnosis of Kindler syndrome. J Dermatol Sci 39:183-185.

Fine JD, Eady RA, Bauer EA, Bauer JW, Bruckner-Tuderman L, Heagerty A, Hintner H, Hovnanian A, Jonkman MF, Leigh I, McGrath JA, Mellerio JE, Murrell DF, Shimizu H, Uitto J, Vahlquist A, Woodley D, Zambruno G. 2008. The classification of inherited epidermolysis bullosa (EB): report of the Third International Consensus Meeting on Diagnosis and Classification of EB. J Am Acad Dermatol 58:931-950.

Glazov EA, Zankl A, Donskoi M, Kenna TJ, Thomas GP, Clark GR, Duncan EL, Brown MA. 2011. Whole-exome re-sequencing in a family quartet identifies POP1 mutations as the cause of a novel skeletal dysplasia. PLoS Genet 7:e1002027.

Goult BT, Bouaouina M, Harburger DS, Bate N, Patel B, Anthis NJ, Campbell ID, Calderwood DA, Barsukov IL, Roberts GC, Critchley DR. 2009. The structure of the N-terminus of kindlin-1: a domain important for alphaiibbeta3 integrin activation. J Mol Biol 394:944-956.

Harburger DS, Bouaouina M, Calderwood DA. 2009. Kindlin-1 and -2 directly bind the C-terminal region of beta integrin cytoplasmic tails and exert integrin-specific activation effects. J Biol Chem 284:11485-114897.

Harburger DS, Calderwood DA. 2009. Integrin signalling at a glance. J Cell Sci 122:159163.

Has C, Herz C, Zimina E, Qu HY, He Y, Zhang ZG, Wen TT, Gache Y, Aumailley M, Bruckner-Tuderman L. 2009. Kindlin-1 is required for RhoGTPase-mediated lamellipodia formation in keratinocytes. Am J Pathol 175:1442-1452.

Has C, Ludwig RJ, Herz C, Kern JS, Ussar S, Ochsendorf FR, Kaufmann R, Schumann H, Kohlhase J, Bruckner-Tuderman L. 2008a. C-terminally truncated kindlin-1 leads to abnormal adhesion and migration of keratinocytes. Br J Dermatol 159:11921196.

Has C, Wessagowit V, Pascucci M, Baer C, Didona B, Wilhelm C, Pedicelli C, Locatelli A, Kohlhase J, Ashton GH, Tadini G, Zambruno G, Bruckner-Tuderman L, McGrath JA, Castiglia D. 2006. Molecular basis of Kindler syndrome in Italy: novel and recurrent Alu/Alu recombination, splice site, nonsense, and frameshift mutations in the KIND1 gene. J Invest Dermatol 126:1776-1783.

Has C, Yordanova I, Balabanova M, Kazandjieva J, Herz C, Kohlhase J, BrucknerTuderman L. 2008b. A novel large FERMT1 (KIND1) gene deletion in Kindler syndrome. J Dermatol Sci 52:209-212.

He Y, Esser P, Heinemann A, Bruckner-Tuderman L, Has C. 2011. Kindlin-1 and -2 have overlapping functions in epithelial cells implications for phenotype modification. Am J Pathol 178:975-982.

Heinemann A, He Y, Zimina E, Boerries M, Busch H, Chmel N, Kurz T, BrucknerTuderman L, Has C. 2011. Induction of phenotype modifying cytokines by FERMT1 mutations. Hum Mutat 32:397-406.

Herz C, Aumailley M, Schulte C, Schlotzer-Schrehardt U, Bruckner-Tuderman L, Has C. 2006. Kindlin-1 is a phosphoprotein involved in regulation of polarity, proliferation, and motility of epidermal keratinocytes. J Biol Chem 281:3608236090 .

Hofmann SC, Voith U, Schonau V, Sorokin L, Bruckner-Tuderman L, Franzke CW. 2009. Plasmin plays a role in the in vitro generation of the linear IgA dermatosis antigen LADB97. J Invest Dermatol 129:1730-1739.

Jobard F, Bouadjar B, Caux F, Hadj-Rabia S, Has C, Matsuda F, Weissenbach J, Lathrop M, Prud'homme JF, Fischer J. 2003. Identification of mutations in a new gene encoding a FERM family protein with a pleckstrin homology domain in Kindler syndrome. Hum Mol Genet 12:925-935.

Kacar N, Semerci N, Ergin S, Pascucci M, Zambruno G, Castiglia D. 2008. A novel frameshift mutation in the KIND1 gene in Turkish siblings with Kindler syndrome. Br J Dermatol 158:1375-1377.

Kern JS, Herz C, Haan E, Moore D, Nottelmann S, von Lilien T, Greiner P, SchmittGraeff A, Opitz OG, Bruckner-Tuderman L, Has C. 2007. Chronic colitis due to an epithelial barrier defect: the role of kindlin-1 isoforms. J Pathol 213:462-470.

Lai-Cheong JE, Liu L, Sethuraman G, Kumar R, Sharma VK, Reddy SR, Vahlquist A, Pather S, Arita K, Wessagowit V, McGrath JA. 2007. Five new homozygous mutations in the KIND1 gene in Kindler syndrome. J Invest Dermatol 127:22682270 .

Lai-Cheong JE, McGrath JA. 2010. Kindler syndrome. Dermatol Clin 28:119-124.

Lai-Cheong JE, Tanaka A, Hawche G, Emanuel P, Maari C, Taskesen M, Akdeniz S, Liu L, McGrath JA. 2009. Kindler syndrome: a focal adhesion genodermatosis. Br J Dermatol 160:233-242. 
Lai-Cheong JE, Wessagowit V, Fassihi H, McGrath JA. 2005. Clinical and molecular aspects of Kindler syndrome. G Ital Dermatol Venereol 140:531538.

Lanschuetzer CM, Muss WH, Emberger M, Pohla-Gubo G, Klausegger A, Bauer JW, Hintner H. 2003. Characteristic immunohistochemical and ultrastructural findings indicate that Kindler's syndrome is an apoptotic skin disorder. J Cutan Pathol 30:553-560.

Larjava H, Plow EF, Wu C. 2008. Kindlins: essential regulators of integrin signalling and cell-matrix adhesion. EMBO Rep 9:1203-1208.

Mansur AT, Elcioglu NH, Aydingoz IE, Akkaya AD, Serdar ZA, Herz C, BrucknerTuderman L, Has C. 2007. Novel and recurrent KIND1 mutations in two patients with Kindler syndrome and severe mucosal involvement. Acta Derm Venereol 87:563-565.

Martignago BC, Lai-Cheong JE, Liu L, McGrath JA, Cestari TF. 2007. Recurrent KIND1 (C20orf42) gene mutation, c.676insC, in a Brazilian pedigree with Kindler syndrome. Br J Dermatol 157:1281-1284.

Moser M, Legate KR, Zent R, Fassler R. 2009. The tail of integrins, talin, and kindlins. Science 324:895-899.

Natsuga K, Nishie W, Shinkuma S, Nakamura H, Matsushima Y, Tatsuta A, Komine M, Shimizu H. 2011. Expression of exon-8-skipped kindlin-1 does not compensate for defects of Kindler syndrome. J Dermatol Sci 61:38-44.

Penagos H, Jaen M, Sancho MT, Saborio MR, Fallas VG, Siegel DH, Frieden IJ. 2004 Kindler syndrome in native Americans from Panama: report of 26 cases. Arch Dermatol 140:939-944

Petricca G, Leppilampi M, Jiang G, Owen GR, Wiebe C, Tu Y, Koivisto L, Hakkinen L, Wu C, Larjava H. 2009. Localization and potential function of kindlin-1 in periodontal tissues. Eur J Oral Sci 117:518-527.
Sadler E, Klausegger A, Muss W, Deinsberger U, Pohla-Gubo G, Laimer M, Lanschuetzer C, Bauer JW, Hintner H. 2006. Novel KIND1 gene mutation in Kindler syndrome with severe gastrointestinal tract involvement. Arch Dermatol 142:1619-1624.

Sethuraman G, Fassihi H, Ashton GH, Bansal A, Kabra M, Sharma VK, McGrath JA. 2005. An Indian child with Kindler syndrome resulting from a new homozygous nonsense mutation (C468X) in the KIND1 gene. Clin Exp Dermatol 30:286-288.

Siegel DH, Ashton GH, Penagos HG, Lee JV, Feiler HS, Wilhelmsen KC, South AP, Smith FJ, Prescott AR, Wessagowit V, Oyama N, Akiyama M, Al Aboud D, Al Aboud K, Al Githami A, Al Hawsawi K, Al Ismaily A, Al-Suwaid R, Atherton DJ, Caputo R, Fine JD, Frieden IJ, Fuchs E, Haber RM, Harada T, Kitajima Y, Mallory SB, Ogawa H, Sahin S, Shimizu H, Suga Y, Tadini G, Tsuchiya K, Wiebe CB, Wojnarowska F, Zaghloul AB, Hamada T, Mallipeddi R, Eady RA, McLean WH, McGrath JA, Epstein EH. 2003. Loss of kindlin-1, a human homolog of the Caenorhabditis elegans actin-extracellular-matrix linker protein UNC-112, causes Kindler syndrome. Am J Hum Genet 73:174-187.

Stang A, Stegmaier C, Jockel KH. 2003. Nonmelanoma skin cancer in the Federal State of Saarland, Germany, 1995-1999. Br J Cancer 89:1205-1208.

Techanukul T, Sethuraman G, Zlotogorski A, Horev L, Macarov M, Trainer A, Fong K, Lens M, Medenica L, Ramesh V, McGrath JA, Lai-Cheong JE. 2011. Novel and recurrent FERMT1 gene mutations in Kindler Syndrome. Acta Derm Venereol 91:267-270.

Ussar S, Moser M, Widmaier M, Rognoni E, Harrer C, Genzel-Boroviczeny O, Fassler R. 2008. Loss of kindlin-1 causes skin atrophy and lethal neonatal intestinal epithelial dysfunction. PLoS Genet 4:e1000289.

Zhou C, Song S, Zhang J. 2009. A novel 3017-bp deletion mutation in the FERMT1 (KIND1) gene in a Chinese family with Kindler syndrome. Br J Dermatol 160:11191122 . 